\title{
Birds of the Grota do Angico Natural Monument in the semi-arid Caatinga scrublands of northeastern Brazil
}

\author{
Juan Ruiz-Esparza ${ }^{1,2,6}$, Sidney Feitosa Gouveia ${ }^{2,3}$, Patricio Adriano da Rocha ${ }^{2,4}$, \\ Raone Beltrão-Mendes ${ }^{2}$, Adauto de Souza Ribeiro ${ }^{5}$ \& Stephen Francis Ferrari ${ }^{5}$ \\ ${ }^{1}$ Graduate Program in Environment and Development, \\ Universidade Federal de Sergipe - UFS, Av. Marechal Rondon s/n, \\ CEP 49100-000, São Cristóvão - Sergipe, Brazil, \\ ${ }^{2}$ Graduate Program in Ecology and Conservation, \\ Universidade Federal de Sergipe - UFS, São Cristóvão - SE, Brazil \\ ${ }^{3}$ Graduate Program in Ecology and Evolution, \\ Universidade Federal de Goiás - UFG, Goiânia - GO, Brazil \\ ${ }^{4}$ Graduate Program in Biological Science (Zoology), \\ Universidade Federal da Paraíba - UFP, João Pessoa - PB, Brazil \\ ${ }^{5}$ Department of Biology, Universidade Federal de Sergipe - SE, São Cristóvão - SE, Brazil \\ ${ }^{6}$ Corresponding author: E-mail: juancolorado21@hotmail.com
}

RUIZ-ESPARZA, J., GOUVEIA, S.F., ROCHA, P.A., BELTRÃO-MENDE S, R., RIBEIRO, A.S., \& FERRARI., S.F. Birds of the Grota do Angico Natural Monument in the semi-arid Caatinga scrublands of northeastern Brazil. Biota Neotrop. 11(2): http://www.biotaneotropica.org.br/v11n2/en/inventory?article+bn01611022011

\begin{abstract}
An inventory of the avian fauna of the Grota do Angico Natural Monument in the Caatinga of northern Sergipe, Brazil, revealed the presence of at least 140 species, including nine that are endemic to the Caatinga and seasonal forest adjacent. Despite the limited scope of the study (two expeditions in July and August, 2008), the species richness recorded at the site appeared to be typical of the region and the Caatinga biome.
\end{abstract}

Keywords: avian inventory, birds conservation, caatinga, protected areas, Sergipe.

RUIZ-ESPARZA, J., GOUVEIA, S.F., ROCHA, P.A., BELTRÃO-MENDE S, R., RIBEIRO, A.S., \& FERRARI., S.F. Aves do Monumento Natural Grota de Angico na região de Caatinga ao Nordeste do Brasil. Biota Neotrop. 11(2): http://www.biotaneotropica.org.br/v11n2/pt/inventory?article+bn01611022011

Resumo: No inventário da avifauna do Monumento Natural Grota de Angico na Caatinga ao norte de Sergipe, Brasil, revelou a presença de pelo menos 140 espécies, incluindo nove endêmicas da Caatinga e florestas sazonais adjacentes. Apesar do alcance limitado do estudo (duas expedições em Julho e Agosto, 2008), a riqueza de espécies registrada no sitio aparentemente pode ser típica da região e do Bioma Caatinga.

Palavras-chave: inventário de aves, conservação de aves, caatinga, unidades de conservação, Sergipe. 


\section{Introduction}

The semi-arid scrublands of the Caatinga cover some $800,000 \mathrm{~km}^{2}$ of the Brazilian Northeast. Relatively few studies of the avian fauna of this biome are available (Pacheco 2000, Olmos et al. 2005), and even basic parameters such as its overall diversity of species are poorlydefined. Estimates of the total number of bird species found in the Caatinga range from 348 (Pacheco 2004) through 460 (Major et al. 2004) to 510 (Silva et al. 2003). It nevertheless seems likely that all of these values represent underestimates, given the paucity of field studies and data.

The Caatinga has suffered extensive anthropogenic degradation, was has formed a landscape characterized by a mosaic of forest fragments and impacted vegetation within a matrix dominated by cattle ranching (Castelleti et al. 2004). This degradation of natural environments generally has deleterious effects on the diversity of a biome (Brooks \& Balmford 1996, Bierregaard Junior et al. 1992), and while the Caatinga has traditionally been considered relatively immune to such impacts, a recent review (Leal et al. 2005) suggests that the Caatinga is as vulnerable as any other biome.

The creation of protected areas is a fundamental step in the conservation of natural resources, and the Sergipe state government has created a number of conservation units since 2007. One of these units is the Grota do Angico Natural Monument, which not only preserves a representative area of arboreal Caatinga on the margin of the São Francisco River, but also has considerable historic and cultural significance as the site of the death of the famous outlaw Virgulino Ferreira da Silva, known as "Lampião".

The aim of the present study was to provide an inventory of the bird species that inhabit a recently-established protected area in the Brazilian Caatinga, and contribute to the understanding of the biological diversity of this biome. As Farias \& Pereira (2009) have pointed out, the avian fauna of the Caatinga is poorly-known, and any addition to the knowledge of biodiversity can provide information to develop strategies for conservation of bird species recorded.

\section{Materials and Methods}

The present study was conducted in the Grota do Angico Natural Monument (GANM), which is located on the right margin of the lower São Francisco River in the northern extreme of the Brazilian state of Sergipe $\left(09^{\circ} 39^{\prime} \mathrm{S} ; 37^{\circ} 40^{\prime} \mathrm{W}\right)$. The 2138 ha reserve is located within the Caatinga morphoclimatic domain, as defined by Ab'Saber (1974), and straddles the border between the municipalities of Poço Redondo and Canindé de São Francisco (Figure 1).

The climate of the region is characterized by relatively reduced and infrequent rainfall, with annual precipitation of approximately $500 \mathrm{~mm}$, distributed mainly between April and August, the period known locally as the winter (inverno). Rainfall is minimal during the remaining months, although the quantity and distribution of precipitation may vary considerably among years. Santos \& Andrade (1992) classified the region as marginally arid (Köppen's BShw category), although local humidity levels may be influenced by the proximity of the area to the São Francisco River (Figure 1). Temperatures are relatively high throughout the year, with means of around $30{ }^{\circ} \mathrm{C}$ during the dry season, but there is also an accentuated diurnal temperature range.

The present study was based on two excursions in 2008, between the $14^{\text {th }}$ and $18^{\text {th }}$ of July, and the $4^{\text {th }}$ and $7^{\text {th }}$ of August, 2008. This period corresponds to the late wet season at the study site.

\section{Mist-netting}

Birds were captured using $100 \mathrm{~m}$-long mist nets with a $35 \mathrm{~mm}$ mesh, which were set along existing trails during morning (5:00-
12:00 AM) and afternoon/evening sessions (4:00-10:00 PM). The specimens were removed carefully from the nets and placed in cloth bags for removal to the field laboratory for processing, where they were identified according to the field guides of Major et al. (2004) and Sigrist (2007), weighed and measured (standard parameters), see Brasil (1994), and then released at the capture sites. Specimen collection was authorized by the Brazilian Environment Institute (IBAMA), through special license number 15900-1, issued by SISBIO. Specimens were collected only when the animal died during capture. All specimens were taxidermized and deposited in the ornithological collection being established at the Federal University of Sergipe in São Cristóvão.

\section{Observation}

Additional records were collected using the active search procedure (Ambrose 1989), for the identification of large, conspicuous or aquatic birds. Observations were conducted using a pair of $8 \times 40$ binoculars, and the field guides of Sigrist (2007) and Major et al. (2004). This procedure was conducted during the earliest daylight hours, when birds tend to be most active. Species nomenclature followed the Brazilian Committee for Ornithological Records (Comitê... 2010).

\section{Results and Discussion}

A total of 140 bird species belonging to 42 families were recorded during the course of the study (Table 1). This total corresponds to almost half (42\%) of the species recorded for the state of Sergipe by Sousa (2009). The Tyrannidae was represented by the largest number of species (17), followed by the Thraupidae (nine species) and the Columbidae, Furnariidae and Emberizidae, each with eight species. Fourteen of the species recorded here are endemic to Brazil, and nine are endemic to the Caatinga (Figure 2), according to Stotz et al. (1996). One of these endemic species, the Pectoral Antwren (Herpsilochmus pectoralis), is listed as vulnerable to extinction by the IUCN (International... 2009).

While July is part of the peak period of bird migrations in the Caatinga (Lima et al. 2003, Ruiz-Esparza 2010), nine migratory species (Zenaida auriculata, Coccyzus melacoryphus, Elaenia chilensis, Euscarthmus meloryphus, Empidonomus varius, Tyrannus melancholicus, Tyrannus savana, Turdus amaurochalinus and Sporophila lineola) was recorded during the present study (based on the classification of Stotz et al. 1996). This single species contrasts with the nine and six migratory species recorded, respectively, in July and August, 2009, at the Serra da Guia, some $40 \mathrm{~km}$ south of the present study site, by (Ruiz-Esparza 2010). While more migratory species were recorded at this site, no waterbirds (e.g. Anhinga anhinga, Egretta thula, Jacana jacana, and Phalacrocorax brasilianus) were observed, presumably because of the absence of aquatic habitats at this higher altitude location. Despite these differences in taxonomic composition, and the much longer study period at Serra da Guia (one year), the two studies recorded the same number of species (140).

Other inventories within the same geographical region have returned similar numbers of taxa. Roos et al. (2006) recorded 145 bird species at the Sobradinho Reservoir (Caatinga), for example, located further upstream on the São Francisco River in Bahia state, whereas D'Horta et al. (2005) recorded 123 species in the Serra de Itabaiana National Park (mixed Caatinga), in southern Sergipe. At Raso da Catarina, Bahia, west and north of the present study site, Lima et al. (2003) recorded a total of 191 bird species in caatinga scrub and semideciduous forest.

Further afield, while the results of some surveys in the Caatinga were similar to those of the present study, others have reported 


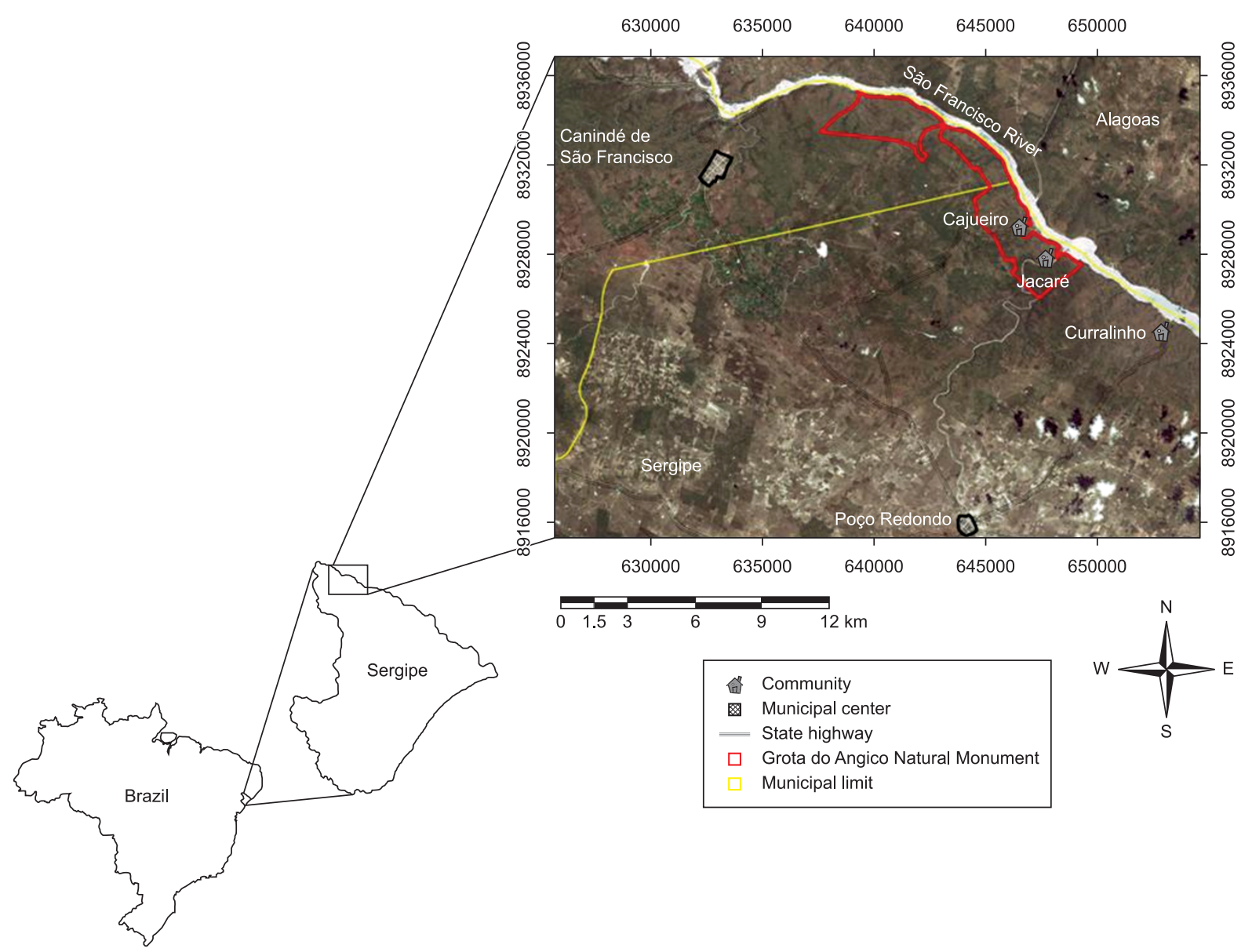

Figure 1. Location of the Grota do Angico Natural Monument in the Brazilian state of Sergipe.

Table 1. Bird species recorded during the present study at the Grota do Angico Natural Monument in northern Sergipe, Brazil. Nomenclature follows the Brazilian Committee for Ornithological Records (Comitê... 2010). $\mathrm{R}=$ resident; $\mathrm{M}=$ migratory; $\mathrm{E}=$ endemic; $\mathrm{SV}=$ seasonal visitor from southern South America; * = specimen collected.

\begin{tabular}{|c|c|c|}
\hline Bird family/species & Common name & Observation \\
\hline \multicolumn{3}{|l|}{ TINAMIDAE (5) } \\
\hline Crypturellus parvirostris (Wagler, 1827) & Small-billed Tinamou & $\mathrm{R}$ \\
\hline Crypturellus tataupa (Temminck, 1815) & Tataupa Tinamou & $\mathrm{R}$ \\
\hline Rhynchotus rufescens (Temminck, 1815) & Red-winged Tinamou & $\mathrm{R}$ \\
\hline Nothura boraquira (Spix, 1825) & White-bellied Nothura & $\mathrm{R}$ \\
\hline Nothura maculosa (Temminck, 1815) & Spotted Nothura & $\mathrm{R}$ \\
\hline \multicolumn{3}{|l|}{ PHALACROCORACIDAE (1) } \\
\hline Phalacrocorax brasilianus (Gmelin, 1789) & Neotropic Cormorant & $\mathrm{R}$ \\
\hline \multicolumn{3}{|l|}{ ANHINGIDAE (1) } \\
\hline Anhinga anhinga (Linnaeus, 1766) & Anhinga & $\mathrm{R}$ \\
\hline \multicolumn{3}{|l|}{ ARDEIDAE (3) } \\
\hline Butorides striata (Linnaeus, 1758) & Striated Heron & $\mathrm{R}$ \\
\hline Bubulcus ibis (Linnaeus, 1758) & Cattle Egret & $\mathrm{R}$ \\
\hline Egretta thula (Molina, 1782) & Snowy Egret & $\mathrm{R}$ \\
\hline \multicolumn{3}{|l|}{ CATHARTIDAE (4) } \\
\hline Cathartes aura (Linnaeus, 1758) & Turkey Vulture & $\mathrm{R}$ \\
\hline Cathartes burrovianus (Cassin, 1845) & Lesser Yellow-headed Vulture & $\mathrm{R}$ \\
\hline Coragyps atratus (Bechstein, 1793) & Black Vulture & $\mathrm{R}$ \\
\hline Sarcoramphus papa (Linnaeus, 1758) & King Vulture & $\mathrm{R}$ \\
\hline \multicolumn{3}{|l|}{ ACCIPITRIDAE (5) } \\
\hline Elanus leucurus (Vieillot, 1818) & White-tailed Kite & $\mathrm{R}$ \\
\hline
\end{tabular}


Table 1. Continued...

\begin{tabular}{|c|c|c|}
\hline Bird family/species & Common name & Observation \\
\hline Geranospiza caerulescens (Vieillot, 1817) & Crane Hawk & $\mathrm{R}$ \\
\hline Heterospizias meridionalis (Latham, 1790) & Savanna Hawk & $\mathrm{R}$ \\
\hline Rupornis magnirostris (Gmelin, 1788) & Roadside Hawk & $\mathrm{R}$ \\
\hline Geranoaetus albicaudatus (Vieillot, 1816) & White-tailed Hawk & $\mathrm{R}$ \\
\hline \multicolumn{3}{|l|}{ FALCONIDAE (4) } \\
\hline Caracara plancus (Miller, 1777) & Southern Caracara & $\mathrm{R}$ \\
\hline Milvago chimachima (Vieillot, 1816) & Yellow-headed Caracara & $\mathrm{R}$ \\
\hline Herpetotheres cachinnans (Linnaeus, 1758) & Laughing Falcon & $\mathrm{R}$ \\
\hline Falco sparverius (Linnaeus, 1758) & American Kestrel & $\mathrm{R}$ \\
\hline \multicolumn{3}{|l|}{ RALLIDAE (1) } \\
\hline Laterallus viridis (Statius Muller, 1776) & Russet-crowned Crake & $\mathrm{R}$ \\
\hline \multicolumn{3}{|l|}{ CARIAMIDAE (1) } \\
\hline Cariama cristata (Linnaeus, 1766) & Red-legged Seriema & $\mathrm{R}$ \\
\hline \multicolumn{3}{|l|}{ CHARADRIIDAE (1) } \\
\hline Vanellus chilensis (Molina, 1782) & Southern Lapwing & $\mathrm{R}$ \\
\hline \multicolumn{3}{|l|}{ RECURVIROSTRIDAE (1) } \\
\hline Himantopus melanurus (Vieillot, 1817) & White-backed Stilt & $\mathrm{R}$ \\
\hline \multicolumn{3}{|l|}{ JACANIDAE (1) } \\
\hline Jacana jacana (Linnaeus, 1766) & Wattled Jacana & $\mathrm{R}$ \\
\hline \multicolumn{3}{|l|}{ COLUMBIDAE (8) } \\
\hline Columbina passerina (Linnaeus, 1758) & Common Ground-Dove & $\mathrm{R}$ \\
\hline Columbina minuta (Linnaeus, 1766) & Plain-breasted Ground-Dove & $\mathrm{R}$ \\
\hline Columbina talpacoti $($ Temminck, 1811$)$ & Ruddy Ground-Dove & $\mathrm{R}$ \\
\hline Columbina squammata (Lesson, 1831) & Scaled Dove & $\mathrm{R}$ \\
\hline Columbina picui* ${ }^{*}$ Temminck, 1813) & Picui Ground-Dove & $\mathrm{R}$ \\
\hline Patagioenas picazuro (Temminck, 1813) & Picazuro Pigeon & $\mathrm{R}$ \\
\hline Zenaida auriculata (Des Murs, 1847) & Eared Dove & M \\
\hline Leptotila verreauxi (Bonaparte, 1855) & White-tipped Dove & $\mathrm{R}$ \\
\hline \multicolumn{3}{|l|}{ PSITTACIDAE (4) } \\
\hline Aratinga aurea (Gmelin, 1788) & Peach-fronted Parakeet & $\mathrm{R}$ \\
\hline Arating a cactorum* ${ }^{*}$ Kuhl, 1820) & Cactus Parakeet & $\mathrm{R}, \mathrm{E}$ \\
\hline Forpus xanthopterygius* (Spix, 1824) & Blue-winged Parrotlet & $\mathrm{R}$ \\
\hline Amazona aestiva (Linnaeus, 1758) & Blue-fronted Parrot & $\mathrm{R}$ \\
\hline \multicolumn{3}{|l|}{ CUCULIDAE (6) } \\
\hline Micrococcyx cinereus (Vieillot, 1817) & Ash-colored Cuckoo & $\mathrm{R}$ \\
\hline Piaya cayana (Linnaeus, 1766) & Squirrel Cuckoo & $\mathrm{R}$ \\
\hline Coccyzus melacoryphus (Vieillot, 1817) & Dark-billed Cuckoo & M \\
\hline Crotophaga ani (Linnaeus, 1758) & Smooth-billed Ani & $\mathrm{R}$ \\
\hline Guira guira (Gmelin, 1788) & Guira Cuckoo & $\mathrm{R}$ \\
\hline Tapera naevia (Linnaeus, 1766) & Striped Cuckoo & $\mathrm{R}$ \\
\hline \multicolumn{3}{|l|}{ TYTONIDAE (1) } \\
\hline Tyto alba (Scopoli, 1769) & Barn Owl & $\mathrm{R}$ \\
\hline \multicolumn{3}{|l|}{ STRIGIDAE (2) } \\
\hline Megascops choliba (Vieillot, 1817) & Tropical Screech-Owl & $\mathrm{R}$ \\
\hline Athene cunicularia (Molina, 1782) & Burrowing Owl & $\mathrm{R}$ \\
\hline \multicolumn{3}{|l|}{ NYCTIBIIDAE (1) } \\
\hline Nyctibius griseus (Gmelin, 1789) & Common Potoo & $\mathrm{R}$ \\
\hline \multicolumn{3}{|l|}{ CAPRIMULGIDAE (4) } \\
\hline Hydropsalis albicollis (Gmelin, 1789) & Pauraque & $\mathrm{R}$ \\
\hline Hydropsalis parvulus (Gould, 1837) & Little Nightjar & $\mathrm{R}$ \\
\hline Hydropsalis hirundinaceus (Spix, 1825) & Pygmy Nightjar & $\mathrm{R}, \mathrm{E}$ \\
\hline Chordeiles pusillus (Gould, 1861) & Least Nighthawk & $\mathrm{R}$ \\
\hline \multicolumn{3}{|l|}{ TROCHILIDAE (4) } \\
\hline Eupetomena macroura (Gmelin, 1788) & Swallow-tailed Hummingbird & $\mathrm{R}$ \\
\hline
\end{tabular}


Table 1. Continued...

\begin{tabular}{|c|c|c|}
\hline Bird family/species & Common name & Observation \\
\hline Chrysolampis mosquitus (Linnaeus, 1758) & Ruby-topaz Hummingbird & $\mathrm{R}$ \\
\hline Chlorostilbon lucidus (Shaw, 1812) & Glittering-bellied Emerald & $\mathrm{R}$ \\
\hline Heliomaster squamosus (Temminck, 1823) & Stripe-breasted Starthroat & $\mathrm{R}, \mathrm{E}$ \\
\hline \multicolumn{3}{|l|}{ ALCEDINIDAE (3) } \\
\hline Megaceryle torquata (Linnaeus, 1766) & Ringed Kingfisher & $\mathrm{R}$ \\
\hline Chloroceryle amazona (Latham, 1790) & Amazon Kingfisher & $\mathrm{R}$ \\
\hline Chloroceryle americana (Gmelin, 1788) & Green Kingfisher & $\mathrm{R}$ \\
\hline \multicolumn{3}{|l|}{ BUCCONIDAE (1) } \\
\hline Nystalus maculatus* (Gmelin, 1788) & Spot-backed Puffbird & $\mathrm{R}$ \\
\hline \multicolumn{3}{|l|}{ PICIDAE (1) } \\
\hline Colaptes campestris (Vieillot, 1818) & Campo Flicker & $\mathrm{R}$ \\
\hline \multicolumn{3}{|l|}{ THAMNOPHILIDAE (6) } \\
\hline Taraba major (Vieillot, 1816) & Great Antshrike & $\mathrm{R}$ \\
\hline Thamnophilus capistratus (Lesson, 1840) & Caatinga Antshrike & $\mathrm{R}, \mathrm{E}$ \\
\hline Thamnophilus pelzelni (Hellmayr, 1924) & Planalto Slaty-Antshrike & $\mathrm{R}, \mathrm{E}$ \\
\hline Myrmorchilus strigilatus (Wied, 1831) & Stripe-backed Antbird & $\mathrm{R}$ \\
\hline Herpsilochmus pectoralis (Sclater, 1857) & Pectoral Antwren & $\mathrm{R}, \mathrm{E}$ \\
\hline Formicivora melanogaster (Pelzeln, 1868) & Black-bellied Antwren & $\mathrm{R}$ \\
\hline \multicolumn{3}{|l|}{ DENDROCOLAPTIDAE (1) } \\
\hline Lepidocolaptes angustirostris (Vieillot, 1818) & Narrow-billed Woodcreeper & $\mathrm{R}$ \\
\hline \multicolumn{3}{|l|}{ FURNARIIDAE (8) } \\
\hline Furnarius leucopus (Swainson, 1838) & Pale-legged Hornero & $\mathrm{R}$ \\
\hline Furnarius rufus (Gmelin, 1788) & Rufous Hornero & $\mathrm{R}$ \\
\hline Synallaxis frontalis (Pelzeln, 1859) & Sooty-fronted Spinetail & $\mathrm{R}$ \\
\hline Synallaxis albescens (Temminck, 1823) & Pale-breasted Spinetail & $\mathrm{R}$ \\
\hline Cranioleuca semicinerea (Reichenbach, 1853) & Gray-headed Spinetail & $\mathrm{R}, \mathrm{E}$ \\
\hline Certhiaxis cinnamomeus (Gmelin, 1788) & Yellow-chinned Spinetail & $\mathrm{R}$ \\
\hline Phacellodomus rufifrons (Wied, 1821) & Rufous-fronted Thornbird & $\mathrm{R}$ \\
\hline Pseudoseisura cristata (Spix, 1824) & Caatinga Cacholote & $\mathrm{R}, \mathrm{E}$ \\
\hline \multicolumn{3}{|l|}{ RHYNCHOCYCLIDAE (3) } \\
\hline Tolmomyias flaviventris (Wied, 1831) & Yellow-breasted Flycatcher & $\mathrm{R}$ \\
\hline Hemitriccus striaticollis (Lafresnaye, 1853) & Stripe-necked Tody-Tyrant & $\mathrm{R}$ \\
\hline $\begin{array}{l}\text { Hemitriccus margaritaceiventer (d'Orbigny \& Lafresnaye, } \\
\text { 1837) }\end{array}$ & Pearly-vented Tody-tyrant & $\mathrm{R}$ \\
\hline \multicolumn{3}{|l|}{ TYRANNIDAE (17) } \\
\hline Myiopagis viridicata (Vieillot, 1817) & Greenish Elaenia & $\mathrm{R}$ \\
\hline Elaenia flavogaster (Thunberg, 1822) & Yellow-bellied Elaenia & $\mathrm{R}$ \\
\hline Elaenia chilensis (Hellmayr, 1927) & Chilean Elaenia & SV \\
\hline Camptostoma obsoletum (Temminck, 1824) & Southern Beardless-Tyrannulet & $\mathrm{R}$ \\
\hline Serpophaga subcristata (Vieillot, 1817) & White-crested Tyrannulet & $\mathrm{R}$ \\
\hline Euscarthmus meloryphus (Wied, 1831) & Tawny-crowned Pygmy-Tyrant & M \\
\hline Sublegatus modestus (Wied, 1831) & Southern Scrub-Flycatcher & $\mathrm{R}$ \\
\hline Fluvicola albiventer (Spix, 1825) & Black-backed Water-Tyrant & $\mathrm{R}$ \\
\hline Fluvicola nengeta (Linnaeus, 1766) & Masked Water-Tyrant & $\mathrm{R}$ \\
\hline Arundinicola leucocephala (Linnaeus, 1764) & White-headed Marsh-Tyrant & $\mathrm{R}$ \\
\hline Machetornis rixosa (Vieillot, 1819) & Cattle Tyrant & $\mathrm{R}$ \\
\hline Pitangus sulphuratus (Linnaeus, 1766) & Great Kiskadee & $\mathrm{R}$ \\
\hline Megarynchus pitangua (Linnaeus, 1766) & Boat-billed Flycatcher & $\mathrm{R}$ \\
\hline Empidonomus varius (Vieillot, 1818) & Variegated Flycatcher & M \\
\hline Tyrannus melancholicus (Vieillot, 1819) & Tropical Kingbird & M \\
\hline Tyrannus savana (Vieillot, 1808) & Fork-tailed Flycatcher & M \\
\hline Myiarchus tyrannulus (Statius Muller, 1776) & Brown-crested Flycatcher & $\mathrm{R}$ \\
\hline \multicolumn{3}{|l|}{ TITYRIDAE (3) } \\
\hline Pachyramphus viridis* (Vieillot, 1816) & Green-backed Becard & $\mathrm{R}$ \\
\hline
\end{tabular}


Table 1. Continued...

\begin{tabular}{|c|c|c|}
\hline Bird family/species & Common name & Observation \\
\hline Pachyramphus marginatus (Lichtenstein, 1823) & Black-capped Becard & $\mathrm{R}$ \\
\hline Pachyramphus validus* (Lichtenstein, 1823) & Crested Becard & $\mathrm{R}$ \\
\hline \multicolumn{3}{|l|}{ VIREONIDAE (2) } \\
\hline Cyclarhis gujanensis (Gmelin, 1789) & Rufous-browed Peppershrike & $\mathrm{R}$ \\
\hline Hylophilus amaurocephalus (Nordmann, 1835) & Gray-eyed Greenlet & $\mathrm{R}, \mathrm{E}$ \\
\hline \multicolumn{3}{|l|}{ CORVIDAE (1) } \\
\hline Cyanocorax cyanopogon (Wied, 1821) & White-naped Jay & $\mathrm{R}, \mathrm{E}$ \\
\hline \multicolumn{3}{|l|}{ HIRUNDINIDAE (1) } \\
\hline Tachycineta leucorrhoa (Vieillot, 1817) & White-rumped Swallow & $\mathrm{R}$ \\
\hline \multicolumn{3}{|l|}{ TROGLODYTIDAE (2) } \\
\hline Troglodytes musculus (Naumann, 1823) & Southern House-Wren & $\mathrm{R}$ \\
\hline Cantorchilus longirostris (Vieillot, 1819) & Long-billed Wren & $\mathrm{R}, \mathrm{E}$ \\
\hline \multicolumn{3}{|l|}{ POLIOPTILIDAE (1) } \\
\hline Polioptila plumbea (Gmelin, 1788) & Tropical Gnatcatcher & $\mathrm{R}$ \\
\hline \multicolumn{3}{|l|}{ TURDIDAE (3) } \\
\hline Turdus rufiventris (Vieillot, 1818) & Rufous-bellied Thrush & $\mathrm{R}$ \\
\hline Turdus leucomelas (Vieillot, 1818) & Pale-breasted Thrush & $\mathrm{R}$ \\
\hline Turdus amaurochalinus* (Cabanis, 1850) & Creamy-bellied Thrush & M \\
\hline \multicolumn{3}{|l|}{ MIMIDAE (1) } \\
\hline Mimus saturninus (Lichtenstein, 1823) & Chalk-browed Mockingbird & $\mathrm{R}$ \\
\hline \multicolumn{3}{|l|}{ COEREBIDAE (1) } \\
\hline Coereba flaveola (Linnaeus, 1758) & Bananaquit & $\mathrm{R}$ \\
\hline \multicolumn{3}{|l|}{ THRAUPIDAE (9) } \\
\hline Saltatricula atricollis (Vieillot, 1817) & Black-throated Saltator & $\mathrm{R}$ \\
\hline Compsothraupis loricata (Lichtenstein, 1819) & Scarlet-throated Tanager & $\mathrm{R}, \mathrm{E}$ \\
\hline Nemosia pileata (Boddaert, 1783) & Hooded Tanager & $\mathrm{R}$ \\
\hline Thlypopsis sordida (d'Orbigny \& Lafresnaye, 1837) & Orange-headed Tanager & $\mathrm{R}$ \\
\hline Tachyphonus rufus (Boddaert, 1783) & White-lined Tanager & $\mathrm{R}$ \\
\hline Lanio pileatus (Wied, 1821) & Pileated-Finch & $\mathrm{R}$ \\
\hline Tangara sayaca (Linnaeus, 1766) & Sayaca Tanager & $\mathrm{R}$ \\
\hline Tangara palmarum (Wied, 1823) & Palm Tanager & $\mathrm{R}$ \\
\hline Paroaria dominicana (Linnaeus, 1758) & Red-cowled Cardinal & $\mathrm{R}, \mathrm{E}$ \\
\hline \multicolumn{3}{|l|}{ EMBERIZIDAE (8) } \\
\hline Zonotrichia capensis (Statius Muller, 1776) & Rufous-collared Sparrow & $\mathrm{R}$ \\
\hline Sicalis flaveola (Linnaeus, 1766) & Saffron Finch & $\mathrm{R}$ \\
\hline Sicalis luteola (Sparrman, 1789) & Grassland Yellow-Finch & $\mathrm{R}$ \\
\hline Emberizoides herbicola (Vieillot, 1817) & Wedge-tailed Grass-Finch & $\mathrm{R}$ \\
\hline Volatinia jacarina (Linnaeus, 1766) & Blue-black Grassquit & $\mathrm{R}$ \\
\hline Sporophila lineola (Linnaeus, 1758) & Lined Seedeater & M \\
\hline Sporophila nigricollis (Vieillot, 1823) & Yellow-bellied Seedeater & $\mathrm{R}$ \\
\hline Sporophila albogularis (Spix, 1825) & White-throated Seedeater & $\mathrm{R}, \mathrm{E}$ \\
\hline \multicolumn{3}{|l|}{ PARULIDAE (1) } \\
\hline Parula pitiayumi (Vieillot, 1817) & Tropical Parula & $\mathrm{R}$ \\
\hline \multicolumn{3}{|l|}{ ICTERIDAE (6) } \\
\hline Icterus cayanensis (Linnaeus, 1766) & Epaulet Oriole & $\mathrm{R}$ \\
\hline Icterus jamacaii (Gmelin, 1788) & Campo Troupial & $\mathrm{R}, \mathrm{E}$ \\
\hline Chrysomus ruficapillus (Vieillot, 1819) & Chestnut-capped Blackbird & $\mathrm{R}$ \\
\hline Agelaioides fringillarius (Vieillot, 1819) & Bay-winged Cowbird & $\mathrm{R}, \mathrm{E}$ \\
\hline Molothrus bonariensis (Gmelin, 1789) & Shiny Cowbird & $\mathrm{R}$ \\
\hline Sturnella militaris (Linnaeus, 1758) & Red-breasted Blackbird & $\mathrm{R}$ \\
\hline \multicolumn{3}{|l|}{ FRINGILLIDAE (2) } \\
\hline Euphonia chlorotica (Linnaeus, 1766) & Purple-throated Euphonia & $\mathrm{R}$ \\
\hline Euphonia violacea (Linnaeus, 1758) & Violaceous Euphonia & $\mathrm{R}$ \\
\hline \multicolumn{3}{|l|}{ PASSERIDAE (1) } \\
\hline Passer domesticus (Linnaeus, 1758) & House Sparrow & $\mathrm{R}$ \\
\hline
\end{tabular}



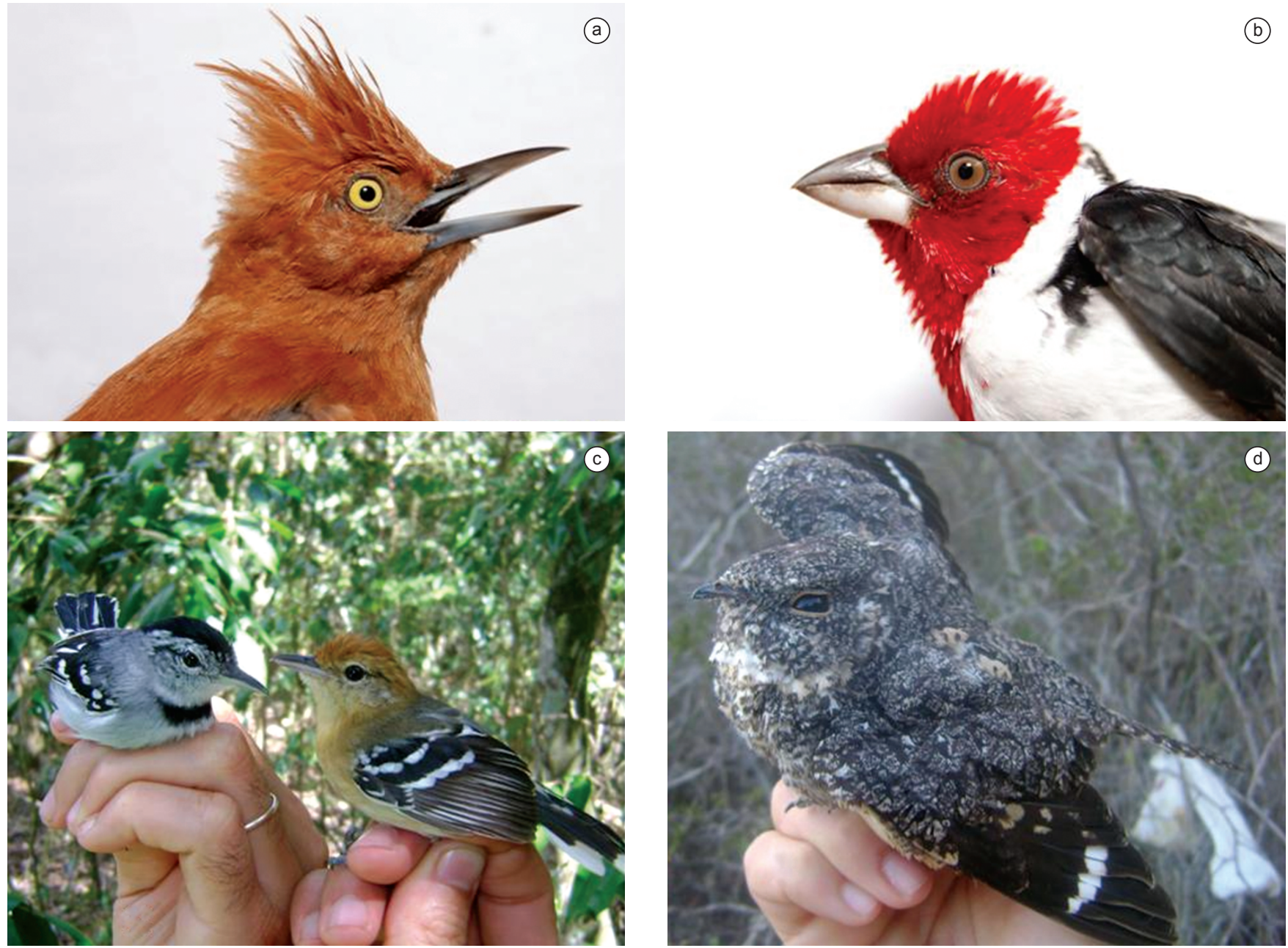

Figure 2. Some species endemic to the Caatinga and seasonal forest adjacent recorded during the present study: a) Pseudoseisura cristata, b) Paroaria dominicana, c) Herpsilochmus pectoralis (male and female), and d) Hydropsalis hirundinaceus.

larger numbers of species. For example, Santos (2004) recorded only 115 bird species in the caatinga of Piauí, while TelinoJúnior et al. (2005) recorded 145 species in Santa Terezinha, Paraíba. Similarly, Nascimento (2000) recorded only 116 species at the Seridó Ecological Station in Rio Grande do Norte, and 154 species at the Ajuba Ecological Station in Ceará, while Farias et al. (2010) found 162 species in the Negreiros National Forest in Pernambuco. By contrast, Nascimento et al. (2000) reported a total of 193 species from the Araripe Plateau, located in the contact zone between the states of Pernambuco, Ceará, and Piauí, Olmos (1993) recorded 208 in the Serra da Capivara National Park in Piauí, and Farias et al. (2006) found a total of 249 species in areas of caatinga habitat in Pernambuco, Paraíba, and Ceará considered to be high priority for conservation.

While it seems likely that the full complement of species found in the Grota do Angico Natural Monument was not recorded here, due primarily to the relatively short duration of the study, this comparison among studies suggests that the estimate of species richness was relatively reliable, by regional standards.

\section{Acknowledgements}

We thank the Sergipe state environment ministry (SEMARH) for logistic support, FAPITEC-Sergipe (JMREA) and CAPES (PAR, RBM) for graduate stipends, and CNPq (project numbers $302747 / 2008-7$ and $476064 / 2008-2$ ). We are especially grateful to
Manoel Messias Nazaré, 'Seu Didi', Eduardo Santos Marques Jr, Crizanto Brito and Evellyn Freitas for their assistance in the field, and to two anonymous reviewers for their helpful comments on the original manuscript.

\section{Referências Bibliográficas}

AB'SABER, A.N. 1974. O domínio morfoclimático semi-árido das Caatingas Brasileiras. Geomorfologia 4:1-39.

AMBROSE, S. 1989. The Australian bird count-Have we got your numbers? RAOU Newsletter 80:1-2.

BIERREGAARD JUNIOR, R.O., LOVEJOY, T.E., KAPOS, V., SANTOS, A.A. \& HUTCHINGS, R.W. 1992. The biological dynamics of tropical rainforest fragments: a prospective comparison of fragments and continuous forest. BioScience 42:859-866.

BRASIL. Ministério do Meio Ambiente e da Amazônia Legal. Instituto Brasileiro do Meio Ambiente e dos Recursos Naturais Renováveis. Centro Nacional de Pesquisas para Conservação das Aves Silvestres - CEMAVE. 1994. Manual de anilhamento de aves silvestres. $2^{\text {nd }} e d$. IBAMA, Brasília, $148 \mathrm{p}$

CASTELLETTI, C.H.M., SILVA, J.M.C., TABARELLI, M. \& SANTOS, A.M.M. 2004. Quanto ainda resta da Caatinga? Uma estimativa preliminar. In Biodiversidade da Caatinga: áreas e ações prioritárias para a conservação. (J.M.C. Silva, M. Tabarelli, M.T. Fonseca \& L.V. Lins, orgs.). Ministério do Meio Ambiente, Brasília, p. 91-100. 
COMITÊ BRASILEIRO DE REGISTROS ORNITOLÓGICOS - CBRO. 2010. Lista das Aves do Brasil. $8^{\text {nd }}$ ed. CBRO, São Paulo. http://www. cbro.org.br (último acesso em 23/12/2010).

D'HORTA, F.M., GOUVEIA, S.F. \& ROCHA, P.A. 2005. Aves. In Parque Nacional Serra de Itabaiana - Levantamento da Biota (Carvalho, C.M. \& Vilar J.C., coord.). Ibama, Biologia Geral e Experimental, Aracaju, p. 63-76.

FARIAS, G.B., GIRÃO E SILVA, W.A. \& ALBANO, C.G. 2006. Diversidade de aves em áreas prioritárias para conservação de aves da Caatinga. In Análise das variações da biodiversidade do bioma Caatinga: suporte a estratégias regionais de conservação (F.S. Araujo, M.J.N. Rodal \& M.R.V. Barbosa). Ministério do Meio Ambiente, Brasília.

FARIAS, G.B. \& PEREIRA, G.P. 2009. Aves de Pernambuco: o estado atual do conhecimento ornitológico. Biotemas 22(3):1-10.

FARIAS, G.B., PEREIRA, G.P. \& BURGOS, K.Q. 2010. Aves da Floresta Nacional de Negreiros (Serrita, Pernambuco). Atual. Ornitol. 157:41-46.

INTERNATIONAL UNION FOR CONSERVATION OF NATURE - IUCN. 2009. IUCN Red List of Threatened Species. Version 2009.1 http://www. iucnredlist.org. (último acesso em 12/12/2009).

LEAL, I.R., SILVA, J.M.C., TABARELLI, M. \& LACHER-JUNIOR, T.E. 2005. Mudando o curso da conservação da biodiversidade na Caatinga do Nordeste do Brasil. Megadiversidade 1:139-146.

LIMA, C.P., SANTOS, S.S. \& LIMA, R.C. 2003. Levantamento e Anilhamento da Ornitofauna na Pátria da Arara-Azul-de-Lear (Anodorhynchus leari, Bonaparte, 1856): um complemento ao Levantamento realizado por $\mathrm{H}$. Sick, L. P. Gonzaga e D. M. Teixeira, 1987. Atual. Ornitol. 112:11-22

MAJOR, I., GONZAGA, S.J.L. \& CASTRO, R. 2004. Aves da Caatinga. Edições Demócrito Rocha, Associação Caatinga, Fortaleza, 256 p.

NASCIMENTO, J.L.X. 2000. Estudo comparativo em duas Estações Ecológicas da Caatinga: Aiuaba e Seridó. Melopsittacus 3:12-35.

NASCIMENTO, J.L.X., NASCIMENTO L.S., AZEVEDO-JÚNIOR, S.M. 2000. Aves da Chapada do Araripe (Brasil): biologia e conservação. Ararajuba 8:115-125.

OLMOS, F. 1993. Birds of Serra da Capivara National Park, in "caatinga" of north-eastern Brazil. Bird Conserv. Int. 3:21-36. http://dx.doi. org/10.1017/S0959270900000769
OLMOS, F., SILVA, W.A.G. \& ALBANO, C.G. 2005. Aves em oito áreas de Caatinga no sul do Ceará e Oeste de Pernambuco, Nordeste do Brasil: composição, riqueza e similaridade. Pap. Avulsos Zool. 45(14):179-199.

PACHECO, J.F. 2000. A ornitologia descobre o sertão: um balanço do conhecimento da avifauna da caatinga dos primórdios aos anos 1950. In Ornitologia Brasileiro no século XX (Straube, F.C., Oliveira, M.M.A. e Cândido-Junior, J.F., eds.). UNISUL/SOB, Curitiba, p 11-70.

PACHECO, J.F. 2004. Aves da Caatinga: uma análise histórica do conhecimento. In Biodiversidade da Caatinga: áreas e ações prioritárias para a conservação (J.M.C. Silva, M. Tabarelli, M.T. Fonseca \& L.V. Lins orgs.). Ministério do Meio Ambiente, Brasília, 189-250 p.

ROOS, A.L., NUNES, M.F.C., SOUSA, E.A., SOUSA, A.E.B.A., NASCIMENTO, J.L.X. \& LACERDA, R.C.A. 2006. Avifauna da região do Lago de Sobradinho: composição, riqueza e biologia. Ornithologia 1(2):135-160.

RUIZ-ESPARZA, A.J.M. 2010. Diversidade da avifauna da serra da Guia, Sergipe e Bahia. Dissertação de Mestrado em Ecologia e Conservação, Universidade Federal de Sergipe, Aracajú, 58 p.

SANTOS, A.F. \& ANDRADE, J.A. 1992. Caracterização e delimitação do semi-árido sergipano. CNPq/UFS, Aracaju.

SANTOS, M.P.D. 2004. As comunidades de aves em duas fisionomias da vegetação da Caatinga no estado do Piauí, Brasil. Ararajuba. 12(2):113-123.

SIGRIST, T. 2007. Guia de campo, aves do Brasil Oriental. Avisbrasilis, São Paulo, 448 p.

SILVA, J.M.C., SOUZA, M.A. BIEBER, A.G.D. \& CARLOS, C.J. 2003. Aves da Caatinga: status, uso do habitat e sensitividade. In Ecologia e conservação da Caatinga (I.R. Leal, M. Tabarelli \& J.M.C. Silva, eds.). Editora Universitária, Universidade Federal de Pernambuco, Recife, p. 237-273.

SOUSA, M.C. 2009. As aves de oito localidades do Estado de Sergipe. Atual. Ornitol. 149:33-57.

STOTZ, D.F., FITZPATRICK, J.W., PARKER III, T.A. \& MOSKOVITS, D.K. 1996. Neotropical Birds Ecology and Conservation. The University of Chicago Press, Chicago, 502 p.

TELINO-JÚNIOR, W.R., LYRA-NEVES, R.M. \& NASCIMENTO, J.L.X. 2005. Biologia e composição da avifauna em uma Reserva Particular de Patrimônio Natural da caatinga paraibana. Ornithologia 1(1):49-57.

Recebido em 26/10/2010

Versão reformulada recebida em 06/01/2011

Publicado em 04/05/2011 